\title{
Caractérisation des gonflements tardifs dans les fromages de St-Nectaire et certaines pâtes pressées
}

\author{
par \\ D. MAYENOBE, R. DIDIENNE et G. PRADEL
}

\section{Résu mé}

Dans l'étude des gonflements tardifs des fromages de SaintNectaire la méthode chimique de caractérisation des germes responsables de cet accident par détermination des A.G.V. (acides gras volatils) a été comparée à la méthode fondée sur le dénombrement microbien. Les données de l'analyse chimique corroborent les données microbiologiques dans la mise en évidence de l'intervention de la fermentation propionique dans le gonflement. Par contre, la mise en évidence du rôle de la flore butyrique ne peut être obtenue que par détermination des A.G.V. dans les cas où le dénombrement des spores ne traduit aucune variation significative.

Le rôle de la fermentation butyrique dans le gonflement du Saint-Nectaire est reconnu si la teneur en acide butyrique est supérieure à $300 \mathrm{mg} / \mathrm{kg}$ de fromage. La méthode de détermination des A.G.V. peut être étendue à d'autres fromages à pâte pressée susceptibles de gonfler, tels que le Pyrénées, le Fontal ou le Gouda. Dans ces fromages plus lipolysés, l'intervention de la fermentation butyrique est établie lorsque la teneur en acide butyrique est supérieure à $500 \mathrm{mg} / \mathrm{kg}$, seuil que nous retiendrons pour les fromages à pâte pressée étudiés.

Dans un fromage non gonflé, le risque d'apparition d'un gonflement provoqué par la fermentation butyrique existe lorsque le rapport $\mathrm{C}_{4} / \mathrm{C}_{6}$ atteint la valeur 4 , il augmente lorsque le rapport s'élève. La détermination de ce rapport constitue donc un moyen de prévision des gonflements.

Mots clés :

Pâtes pressées - Gonflements tardifs - Acides gras volatils.

Laboratoire de Recherches Fromagères, I.N.R.A., rue de Salers - 15000 Aurillac (France). 
Titre abrégé :

Gonflements tardifs dans le fromage de St-Nectaire.

\section{Su $\mathrm{m}$ m a r y}

\section{CHARACTERIZATION OF THE LATE BLOWING IN ST-NECTAIRE CHEESE AND SOME PRESSED CHEESE}

In the study of the late blowing defect of Saint Nectaire cheese characterization of the microorganisms responsible by determination of the V.F.A. (volatile fatty acids) was compared with the method based on enumeration procedure.

In case of late blowing caused by propionic fermentation data from chemical determination correlated well with those from microorganisms enumeration. On the other hand characterization of butyric fermentation responsability from V.F.A. determinations was obtained in cases in which no significant variation appeared from spore enumeration.

Butyric fermentation responsability in late blowing defect of Saint Nectaire cheese was proved when butyric acid concentration in cheese was higher than $300 \mathrm{mg} / \mathrm{kg}$ of cheese. Utilization of the V.F.A. determination method can be extended to several hard and semi-hard pressed cheese susceptible to late blowing, like Pyrenees, Fontal and Gouda cheese. In more lipolysed cheeses responsability of butyric fermentation is established when butyric acid content exceeded $500 \mathrm{mg} / \mathrm{kg}$ which is the value adopted for hard pressed cheese studied.

In a cheese without late blowing defect risk due to butyric fermentation appears when ratio $C_{4} / C_{6}$ reaches the value 4 . Risk increases as the ratio increases. The determination of this ratio must be considered as a means for predicting late blowing originated by the butyric fermentation.

Key words :

Pressed cheese - Late blowing - Volatile fatty acids.

\section{INTRODUCTION}

Au cours du deuxième mois de l'affinage des fromages de SaintNectaire, des défauts caractérisés par une ouverture trop importante allant parfois jusqu'à l'éclatement peuvent apparaître. Ces gonflements sont qualifiés de tardifs par opposition à ceux qui apparaissent plus tôt au cours de la fabrication. Ils ont pour origine la production de gaz carbonique et d'hydrogène au cours de la fermentation du lactate qui donne lieu par ailleurs à formation des acides gras volatils; acides propionique et butyrique. 
La recherche des micro-organismes responsables des gonflements tardifs selon la méthode du dénombrement microbien ne va pas sans difficulté : les formes décelables des bactéries peuvent disparaître après avoir provoqué le défaut; en outre leur dénombrement peut être rendu difficile par la présence d'autres microorganismes, les milieux disponibles n'étant pas suffisamment sélectifs. C'est le cas en particulier des Clostridium tels que C. tyrobutyricum pour lequel le dénombrement ne prend en compte que les formes sporulées et non leurs formes végétatives actives. Le nombre de spores peut ne pas être suffisamment élevé pour qu'on puisse en déduire que cette bactérie s'est multipliée dans le fromage et qu'elle est par conséquent à l'origine du gonflement observé.

Face aux difficultés de mise en œuvre des méthodes fondées sur le dénombrement microbien, nous avons voulu montrer que la détermination des A.G.V. (Acides Gras Volatils) déjà entreprise sur l'Emmental (Kiermeier et al., 1968), marquait un progrès important dans la caractérisation des germes responsables des gonflements tardifs des fromages de Saint-Nectaire.

\section{MATERIEL ET METHODES}

\section{Méthodes chimiques}

\subsection{Extraction des A.G.V. des fromages}

La méthode d'extraction utilisée est celle décrite par Ritter et Hänni, 1960. Vingt à $25 \mathrm{~g}$ de fromage sont pesés puis broyés avec un mixer dans $100 \mathrm{ml}$ d'eau distillée auxquels ont été ajoutées quelques gouttes d'antimousse 426 Rhodorsil. Les A.G.V. sont entraînés par la vapeur dans un distillateur Büchi jusqu'à l'obtention de $500 \mathrm{ml}$ de distillat. Ils sont saponifiés par addition d'une solution de soude $0,1 \mathrm{~N}$ avec ajustement du $\mathrm{pH}$ à 10 .

\subsection{Dosage Des A.G.V.}

Une partie aliquote de $25 \mathrm{ml}$ du distillat est évaporée à siccité dans une étuve à $40^{\circ} \mathrm{C}$. Les savons sont repris dans $2 \mathrm{ml}$ d'eau distillée, et les A.G.V. sont libérés par addition de $10 \mathrm{mg}$ d'acide orthophosphorique. Après addition de $1 \mathrm{mg}$ d'acide valérique, utilisé comme étalon interne de chromatographie, $1 \mu \mathrm{l}$ de la solution d'A.G.V. est injecté dans le chromatographe Girdel 3000. La séparation des A.G.V. est effectuée sur une colonne en acier inoxydable de longueur $2 \mathrm{~m}$ et de diamètre intérieur $2,2 \mathrm{~mm}$, chargée avec du Chromosorb W A W 80-100 imprégné de 10\% de Sp 1200 (Supelco) acidifié par $2 \%$ d'acide phosphorique. Afin d'éliminer les phénomènes de traînées dus principalement à l'adsorption des acides, il est procédé à des injections d'une solution d'acide formique (Ackman et Burgher, 
1963 ; Cochrane, 1976) ou d'une solution d'acide phosphorique $20 \%$ $(\mathrm{v} / \mathrm{v})$ préalablement aux injections d'échantillons.

\section{Méthodes bactériologiques}

\subsection{PRÉparation des dilutions}

Dix grammes de fromage sont mis en suspension dans $90 \mathrm{ml}$ d'une solution de citrate de sodium à $2 \%(\mathrm{p} / \mathrm{v})$. Les dilutions décimales sont ensuite effectuées dans de l'eau peptonée à $0,1 \%$ stérile.

\subsection{DÉNOMBREMENT MICROBIEN}

\subsubsection{Spores de clostridies butyriques}

Les spores de clostridies butyriques sont dénombrées sur le milieu au lactate décrit par Cerf et Bergère, 1968 (milieu pour Clostridium tyrobutyricum Merck $\mathrm{n}^{\circ}$ 11734). Immédiatement avant utilisation, les tubes de milieu sont chauffés à $100^{\circ} \mathrm{C}$ pendant $20 \mathrm{~min}$ pour en chasser l'air, ils sont ensuite refroidis à $45^{\circ} \mathrm{C}$ et ensemencés avec les dilutions (3 tubes par dilution). Dans le but de constituer un bouchon anaérobie au-dessus du milieu, on coule ensuite environ $3 \mathrm{ml}$ d'un mélange en partie égale de paraffine et d'huile de vaseline fondu et ramené à $55^{\circ} \mathrm{C}$. Ce mélange a été stérilisé au préalable à l'autoclave pendant $20 \mathrm{~min}$ à $120^{\circ} \mathrm{C}$. Les tubes ensemencés sont ensuite chauffés à $75^{\circ} \mathrm{C}$ pendant $10 \mathrm{~min}$ et incubés à $37^{\circ} \mathrm{C}$ pendant 8 jours. A partir du nombre de tubes positifs (tubes dont le bouchon de paraffine est soulevé par la production de gaz) est calculé le nombre le plus probable (N.P.P.) de micro-organismes en utilisant la table de Mac Crady, 1918.

\subsubsection{Bactéries propioniques}

Les bactéries propioniques sont dénombrées sur le milieu Y.E.L. (Yeast Extract Sodium Lactate) décrit par Malik et al., 1968. Les milieux ensemencés, coulés dans des tubes de $8 \times 400 \mathrm{~mm}$, sont incubés à $30^{\circ} \mathrm{C}$ pendant 10 jours. On dénombre les colonies lenticulaires catalase positive comme étant des bactéries propioniques dans les fromages contenant moins de 1000 bactéries coliformes par gramme. Dans les fromages renfermant plus de 1000 bactéries coliformes par gramme, des épreuves de confirmation consistant en un repiquage d'un nombre représentatif de colonies catalase positive sur milieu au lactate sont effectuées.

\section{RESULTATS ET DISCUSSION}

\section{Résultats comparés des méthodes chimiques et bactériologiques}

La présentation des résultats des analyses chimiques et bactériologiques réalisées sur des fromages de Saint-Nectaire gonflés 
(tab. 1) fait apparaître différentes situations. Dans un premier cas, celui des échantillons 3 et 4 , le gonflement se traduisait en premier lieu par une forte teneur en acide propionique associée à un nombre élevé de bactéries propioniques, en second lieu par une teneur moyenne en acide butyrique qui n'était accompagnée d'un nombre élevé de spores butyriques que dans l'échantillon 3 seulement. Dans un second cas, celui des échantillons 5 et 6 , le gonflement était marqué par une forte teneur en acide butyrique qui n'était associée à un nombre élevé de spores butyriques que dans l'échantillon 6 seulement. La teneur en acide propionique et le nombre de bactéries propioniques étaient faibles dans l'échantillon 5, plus élevés dans l'échantillon 6. Enfin, dans un troisième cas, échantillons 7 à 10, le gonflement était accompagné de fortes teneurs en acides propionique et butyrique auxquelles correspondait un nombre élevé de bactéries propioniques dans tous les échantillons mais auxquelles ne correspondait un nombre élevé de spores butyriques que dans l'échantillon 10 seulement.

L'interprétation de ces résultats doit prendre en compte les origines respectives des acides gras considérés (Kuzdzal-Savoie et Kuzdzal, 1971 ; Kuzdzal et al., 1971). Dans le fromage de Saint-Nectaire les acides propionique et butyrique sont produits conjointement avec le gaz carbonique et l'hydrogène lors de l'installation accidentelle des fermentations propionique et butyrique. L'acide butyrique a cependant une autre origine non fermentaire dans la lipolyse qui est également responsable de la libération d'acide caproïque. Quant aux acides isobutyrique et isovalérique, ils sont issus de la dégradation des acides aminés par la flore normale et par la flore de contamination.

Les teneurs en acide butyrique des fromages témoins non gonflés (tab. 1) étaient celles de fromages affinés. Les analyses effectuées au Laboratoire sur des fromages de Saint-Nectaire à différents stades d'affinage (fig. 1) font apparaître que dans un fromage non gonflé où cet acide est d'origine lipolytique sa teneur ne dépasse jamais $300 \mathrm{mg} / \mathrm{kg}$ de fromage. Les teneurs plus élevées ne pourront être atteintes que par les interventions conjuguées de la lipolyse et de la fermentation butyrique.

A l'opposé, nous avons observé que dans un fromage gonflé dont la teneur en acide butyrique était inférieure à $300 \mathrm{mg} / \mathrm{kg}$ la fermentation butyrique n'était pas impliquée dans le déclenchement de l'accident.

La teneur en acide propionique aussi bien que le nombre de bactéries propioniques désignaient dans le premier cas la fermentation propionique comme responsable du gonflement (échantillons 3 et 4). Dans le deuxième et le troisième cas (échantillons 5 à 10) la teneur en acide butyrique supérieure à $300 \mathrm{mg}$ mettait en cause la fermentation butyrique dans l'apparition de l'accident. En outre dans le troisième cas (échantillons 7 à 10), les teneurs en acide pro- 
TABLEAU 1 - TABLE 1

Niveaux comparés des acides gras volatils et des flores propionique et butyrique dans les fromages de Saint-Nectaire présentant ou non des défauts de gonflement tardif

Comparative levels of volatile fatty acids and propionic and butyric acid flora in Saint-Nectaire cheese exhibiting or no late blowing fault

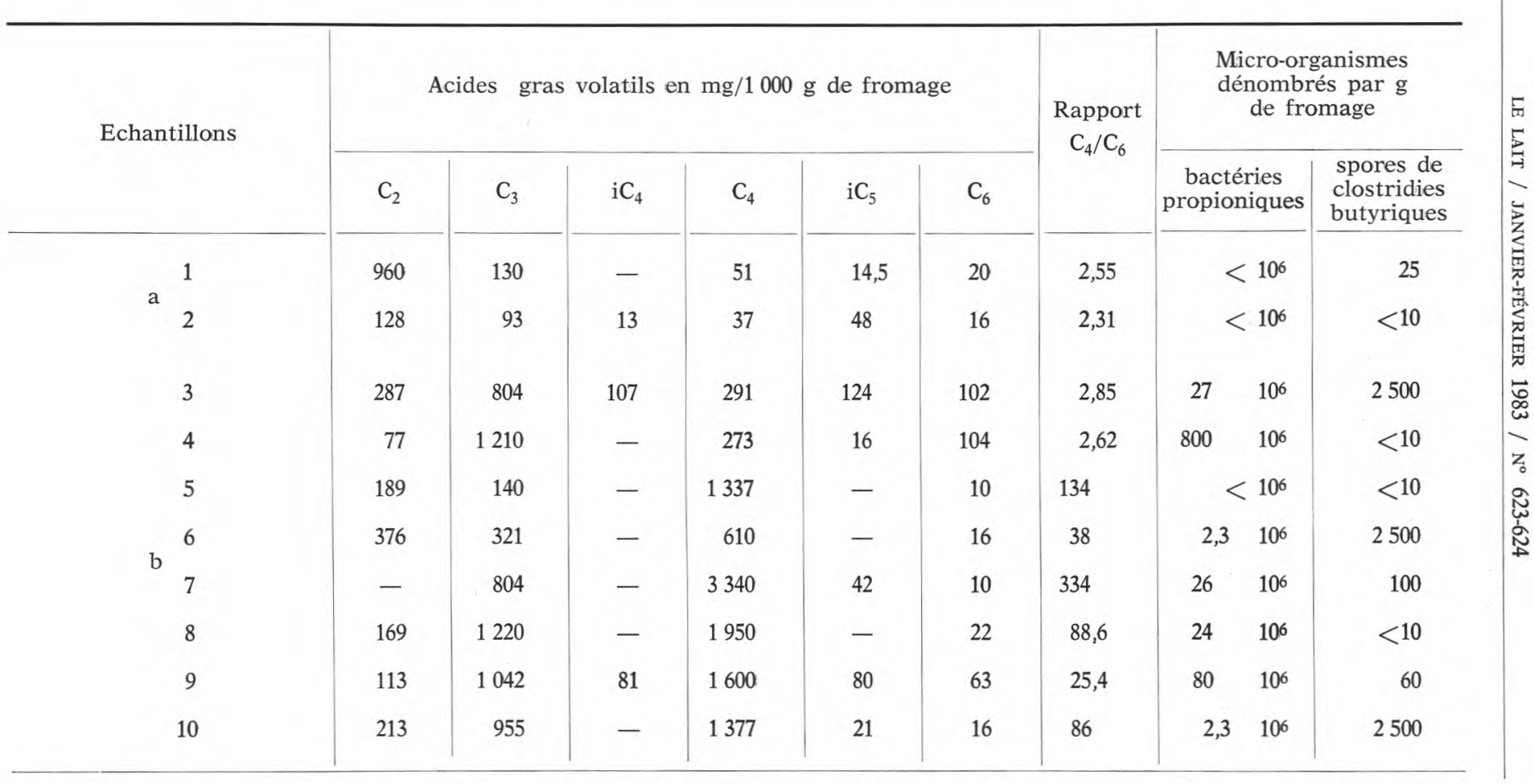

a : Fromages affinés servant de témoins, origine commerciale ; b : Fromages affinés présentant des gonflements tardifs. 


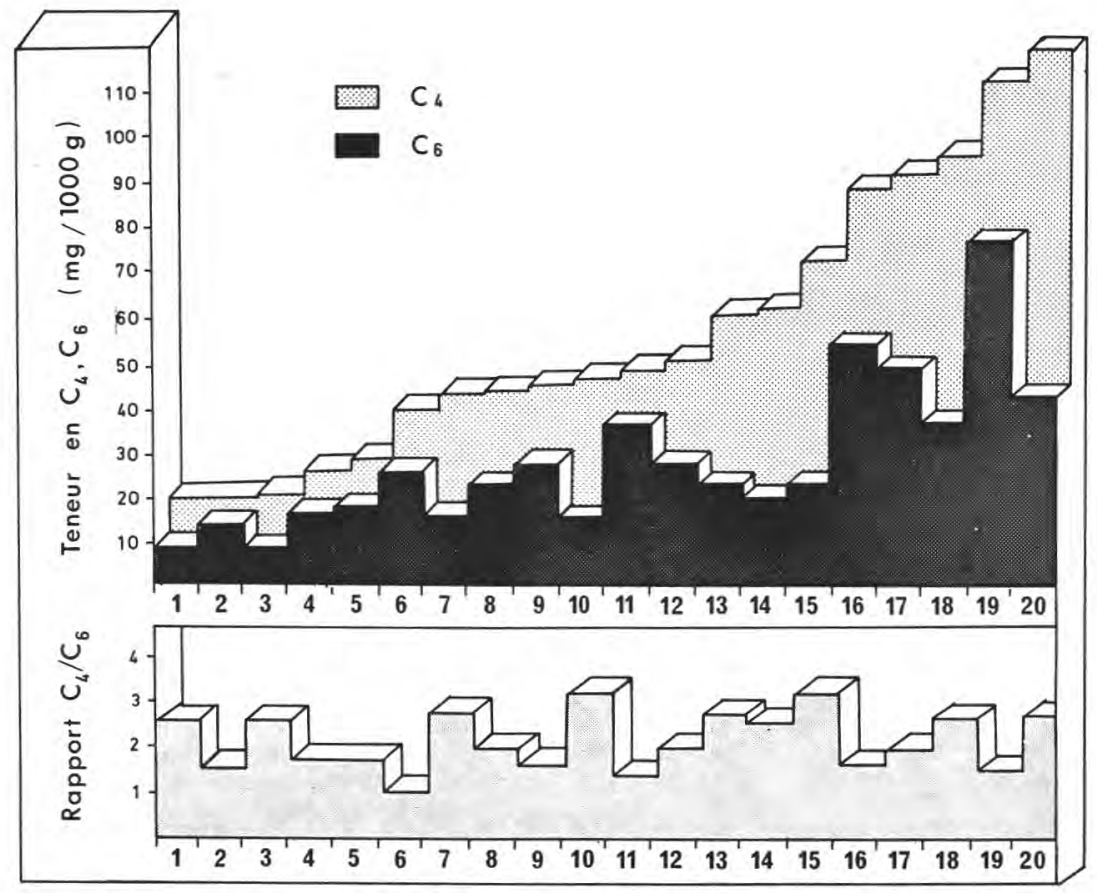

Fig. 1

Teneurs en acide butyrique $\left(\mathrm{C}_{4}\right)$ et caproïque $\left(\mathrm{C}_{6}\right)$ et leur rapport $\mathrm{C}_{4} / \mathrm{C}_{6}$ dans 20 Saint-Nectaire à différents stades d'affinage ne présentant pas de défauts de gonflement.

Butyric $\left(C_{4}\right)$ and caproïc $\left(C_{6}\right)$ acid concentrations and their ratio $C_{4} / C_{6}$ in 20 Saint-Nectaire cheese without blowing fault at various ripening stages.

pionique et le nombre de bactéries propioniques indiquaient une cause secondaire de gonflement dans la fermentation propionique.

Le démarrage de la fermentation butyrique même s'il ne se note pas par l'apparition immédiate de gonflements pourra être décelé au travers des valeurs du rapport $\mathrm{C}_{4} / \mathrm{C}_{6}$ entre la teneur en acide butyrique dans le fromage et la teneur en acide caproïque, ce dernier acide ayant été reconnu comme un bon témoin de la lipolyse car libéré non préférentiellement (Kuzdzal-Savoie et Kuzdzal, 1971). Les résultats de l'analyse de 20 fromages de Saint-Nectaire, d'origines diverses, d'âges différents (fig. 1) montrent que dans ces fromages normaux le rapport $\mathrm{C}_{4} / \mathrm{C}_{6}$ ne dépasse jamais la valeur 4 . Cette valeur sera très rapidement dépassée lorsque de l'acide butyrique d'origine fermentaire viendra s'ajouter à l'acide butyrique issu de la lipolyse. La détermination du rapport $\mathrm{C}_{4} / \mathrm{C}_{6}$ dans un fromage 
TABLEAU $2-T A B L E 2$

Quantités d'acides gras volatils dans les fromages à pâte pressée présentant ou non des défauts de gonflement tardif Quantitative determination of volatile fatty acids in several hard and semi-hard pressed cheese exhibiting or no late blowing fault

\begin{tabular}{|c|c|c|c|c|c|c|c|c|c|c|}
\hline & & & & \multicolumn{6}{|c|}{ Acides gras volatils en $\mathrm{mg} / 1000 \mathrm{~g}$ de fromage } & \multirow{2}{*}{$\begin{array}{c}\text { Rapport } \\
\mathrm{C}_{4} / \mathrm{C}_{6}\end{array}$} \\
\hline \multicolumn{4}{|c|}{ Echantillons } & & & & & & & \\
\hline Types Pyrénées & & $\begin{array}{l}\text { gonflé } \\
\text { gonflé } \\
\text { gonflé } \\
\text { Témoin }\end{array}$ & $\begin{array}{l}1 \\
2 \\
3 \\
4\end{array}$ & $\begin{array}{r}15 \\
145 \\
760 \\
831\end{array}$ & $\begin{array}{r}32 \\
137 \\
- \\
-\end{array}$ & $\begin{array}{l}\overline{-} \\
\overline{2,2}\end{array}$ & $\begin{array}{r}1200 \\
1517 \\
700 \\
24\end{array}$ & $\frac{-}{\overline{8}}$ & $\begin{array}{l}10 \\
10 \\
10 \\
18,5\end{array}$ & $\begin{array}{c}120 \\
152 \\
70 \\
1,3\end{array}$ \\
\hline Type Fontal & & $\begin{array}{l}\text { gonflé } \\
\text { gonflé } \\
\text { gonflé } \\
\text { Témoin }\end{array}$ & $\begin{array}{l}5 \\
6 \\
7 \\
8\end{array}$ & $\begin{array}{l}483 \\
203 \\
140 \\
421\end{array}$ & $\begin{array}{l}-\overline{381} \\
224 \\
-\end{array}$ & $\begin{array}{l}\overline{-} \\
\overline{-}\end{array}$ & $\begin{array}{r}436 \\
2780 \\
2250 \\
4,9\end{array}$ & $\begin{array}{l}\bar{z} \\
\overline{0,3}\end{array}$ & $\begin{array}{l}10 \\
35 \\
10 \\
3,1\end{array}$ & $\begin{array}{c}44 \\
79,4 \\
225 \\
1,6\end{array}$ \\
\hline Type Gouda & non & $\begin{array}{l}\text { gonflé } \\
\text { gonflé } \\
\text { gonflé } \\
\text { Témoin }\end{array}$ & $\begin{array}{r}9 \\
10 \\
11 \\
12\end{array}$ & $\begin{array}{r}41 \\
20 \\
558 \\
663\end{array}$ & $\begin{array}{l}58 \\
99 \\
80 \\
-\end{array}$ & $\frac{-}{248}$ & $\begin{array}{r}750 \\
1704 \\
240 \\
79\end{array}$ & $\frac{-}{20,3}$ & $\begin{array}{l}10 \\
10 \\
11,6 \\
44\end{array}$ & $\begin{array}{c}75 \\
170 \\
20,7 \\
1,8\end{array}$ \\
\hline
\end{tabular}


en cours d'affinage non gonflé renseignera sur le déclenchement et la progression de la fermentation butyrique.

Les données de l'analyse chimique étaient corroborées par les données microbiologiques dans le cas de gonflements ayant pour origine la fermentation propionique. Les paramètres, teneur en acide propionique et nombre de bactéries propioniques sont en relation avec l'activité métabolique des bactéries propioniques. Il n'en est pas de même dans le cas de gonflements ayant pour cause la fermentation butyrique. Les formes actives des clostridies butyriques responsables de la production d'acide butyrique et de gaz ne sont pas concernées par la numération qui ne considère que les spores. Aussi bien la détermination des teneurs en acide butyrique permettait elle, de conclure à l'intervention de la fermentation butyrique dans les échantillons 5, 8 et 9 alors que les numérations de spores restaient à un bas niveau.

\section{Domaine d'application de la méthode de détermination des A.G.V.}

Le tableau 2 rapporte les déterminations d'A.G.V. effectuées sur d'autres fromages à pâte pressée tels le Pyrénées, le Fontal et le Gouda présentant ou non le défaut de gonflement. Dans les échantillons 1, 2, 3, 6, 7, 9 et 10 le niveau élevé en acide butyrique désignait la fermentation butyrique comme responsable du gonflement. Des analyses effectuées au Laboratoire sur divers échantillons de ces 3 types de fromage il ressort en effet que dans le cas où, seule la lipolyse est en cause, la teneur en acide butyrique dans ces fromages ne dépasse pas $500 \mathrm{mg} / \mathrm{kg}$ de fromage. Nous avons noté par ailleurs, que dans un fromage à pâte pressée gonflé ayant une teneur en $\mathrm{C}_{4}$ inférieure à $500 \mathrm{mg} / \mathrm{kg}$, l'intervention de la fermentation butyrique ne pouvait être invoquée comme responsable du gonflement, cas de l'échantillon $\mathrm{n}^{\circ} 5$.

Comme dans le Saint-Nectaire, l'installation de la fermentation butyrique dans un fromage jeune susceptible de gonfler appartenant à l'un de ces 3 types de pâte pressée sera signalée, pour les teneurs en acide butyrique inférieures à $500 \mathrm{mg} / \mathrm{kg}$ lorsque le rapport $\mathrm{C}_{4} / \mathrm{C}_{6}$ prendra des valeurs supérieures à 4 . C'est le cas de l'échantillon 11, tableau 2 , où la valeur du rapport $C_{4} / C_{6}$ traduit l'existence de la fermentation butyrique dans un fromage apparemment sain.

\section{CONCLUSIONS}

La détermination des A.G.V. dans les fromages de Saint-Nectaire met bien en évidence la double origine, fermentations propionique et butyrique, des gonflements tardifs de ces fromages. Le développement de la fermentation propionique à un faible niveau est d'ail- 
leurs souhaité; il donne au fromage de Saint-Nectaire une légère ouverture de 3 à 4 trous, élément de typage par rapport à d'autres fromages à pâte pressée demi-ferme. Cependant un excès de fermentation propionique entraînant un gonflement du fromage $\left(\mathrm{C}_{3}>\right.$ à $500 \mathrm{mg} / \mathrm{kg}$ ), seul ou en association avec la fermentation butyrique, peut être considéré comme un défaut.

Eu égard aux valeurs élevées atteintes par les teneurs en acide butyrique dans les fromages ayant subi un gonflement, le seuil absolu d'une teneur en $\mathrm{C}_{4}$ égale à $500 \mathrm{mg} / \mathrm{kg}$ à partir duquel l'intervention de la fermentation butyrique est certaine, pourrait être retenu pour les fromages à pâtes pressées susceptibles de présenter ce type de gonflement tardif.

La méthode de détermination des A.G.V., si elle rend plus aisée la caractérisation des gonflements tardifs, en facilite également la prévision en cours d'affinage. Lorsqu'un fromage jeune lors d'une première analyse aura un rapport $C_{4} / C_{6}$ supérieur à 4 , un risque de gonflement existera. Si une analyse postérieure fait apparaître une augmentation $\mathrm{du}$ rapport $\mathrm{C}_{4} / \mathrm{C}_{6}$ le risque se précisera.

Ainsi cette méthode d'une mise en œuvre simple pourrait-elle être intégrée dans les opérations de contrôle des fabrications présentant un haut risque de gonflement, dès le second mois d'affinage.

\section{Bibliographie}

ACKMAN (R. G.) and BURgHeR (R. D.) (1963). - Quantitative gas liquid chromatographic estimation of volatile fatty acids in aqueous media. Anal. Chem., 35 (6), 647-652.

CERF (O.) et Bergère (J. L.) (1968). - La numération des spores de Clostridium et son application au lait et aux produits laitiers. II. Numération des différents groupes de Clostridium. Le Lait, 478, 501-519.

Cochrane (G. C.) (1975), - A review of the analysis of free fatty acids $\left(\mathrm{C}_{2}-\mathrm{C}_{6}\right)$. J. Chromatog. Sci., 13, 440-447.

Kiermeier (F.), MAYr (A.) und Hanusch (J.) (1968), - Zur Entstehung flüchtiger Fettsäuren bei der Propionsäure- und Buttersäuregärung im Emmentaler Käse. II. Einflu $\beta$ der Buttersäuregärung. Z. Lebensmitt. Untersuch. Forsch., 137, 273-282.

Kuzdzal-Savoie (S.) et Kuzdzal (W.) (1971). - Dosage des acides gras libres dans quelques fromages français. La Technique Laitière, 717, 11-13.

Kuzdzal-Savore (S.), Kuzdzal (W.), Goto (K.) et Trehin (J.) (1971). - Les acides gras libres du Munster. Revue Laitière Française, 290, 615-629.

MAC CRADY (M. H.) (1918). - Tables for rapid interpretation of fermentation tube results. Can. publ. Hith., 5, 9, 201-210.

Malik (A. C.), Reinbold (G. W.) and Vedamuthu (E. R.) (1968). - An evaluation of the taxonomy of Propionibacterium. Can. J. Microbiol., 14, 1185-1191.

Ritter (W.) und Hanni (H.) (1960). - Die Anwendung der Gaschromatographie in der Milchwirtschaft. I. Der Nachweis und die Bestimmung der flüichtigen Fettsäuren in Milchprodukten und Kulturen. Milchwissenschaft, 15 (6), 296-302. 